\title{
MADIBA: A web server toolkit for biological interpretation of Plasmodium and plant gene clusters
} Philip J Law ${ }^{\dagger 1}$, Clotilde Claudel-Renard ${ }^{\dagger 1,3}$, Fourie Joubert ${ }^{1}$, Abraham I Louw ${ }^{1}$ and Dave K Berger*2

\begin{abstract}
Address: ${ }^{1 B i o i n f o r m a t i c s ~ a n d ~ C o m p u t a t i o n a l ~ B i o l o g y ~ U n i t, ~ A f r i c a n ~ C e n t r e ~ f o r ~ G e n e ~ T e c h n o l o g i e s ~(A C G T), ~ D e p a r t m e n t ~ o f ~ B i o c h e m i s t r y, ~ F a c u l t y ~}$ of Natural and Agricultural Sciences, University of Pretoria, Pretoria, 0002, South Africa, ${ }^{2}$ Department of Plant Science, Forestry and Agricultural Biotechnology Institute (FABI), University of Pretoria, Pretoria, 0002, South Africa and ${ }^{3}$ Laboratoire de Biologie Cellulaire et Moléculaire, Biogemma, Domaine de Sandreau, 31700, Mondonville, France

Email: Philip J Law - plaw@tuks.co.za; Clotilde Claudel-Renard - clotilde.claudel@biogemma.fr; Fourie Joubert - fjoubert@postino.up.ac.za; Abraham I Louw - Braam.Louw@up.ac.za; Dave K Berger* - dave.berger@up.ac.za

* Corresponding author †Equal contributors
\end{abstract}

Published: 28 February 2008

BMC Genomics 2008, 9:105 doi:10.1186/147|-2164-9-105
Received: 21 October 2007

Accepted: 28 February 2008

This article is available from: http://www.biomedcentral.com/I47I-2/64/9//05

(C) 2008 Law et al; licensee BioMed Central Ltd.

This is an Open Access article distributed under the terms of the Creative Commons Attribution License (http://creativecommons.org/licenses/by/2.0), which permits unrestricted use, distribution, and reproduction in any medium, provided the original work is properly cited.

\begin{abstract}
Background: Microarray technology makes it possible to identify changes in gene expression of an organism, under various conditions. Data mining is thus essential for deducing significant biological information such as the identification of new biological mechanisms or putative drug targets. While many algorithms and software have been developed for analysing gene expression, the extraction of relevant information from experimental data is still a substantial challenge, requiring significant time and skill.
\end{abstract}

Description: MADIBA (MicroArray Data Interface for Biological Annotation) facilitates the assignment of biological meaning to gene expression clusters by automating the post-processing stage. A relational database has been designed to store the data from gene to pathway for Plasmodium, rice and Arabidopsis. Tools within the web interface allow rapid analyses for the identification of the Gene Ontology terms relevant to each cluster; visualising the metabolic pathways where the genes are implicated, their genomic localisations, putative common transcriptional regulatory elements in the upstream sequences, and an analysis specific to the organism being studied.

Conclusion: MADIBA is an integrated, online tool that will assist researchers in interpreting their results and understand the meaning of the co-expression of a cluster of genes. Functionality of MADIBA was validated by analysing a number of gene clusters from several published experiments - expression profiling of the Plasmodium life cycle, and salt stress treatments of Arabidopsis and rice. In most of the cases, the same conclusions found by the authors were quickly and easily obtained after analysing the gene clusters with MADIBA.

\section{Background}

A greater understanding of the biological mechanisms within organisms becomes possible with the availability of complete genome data, in combination with highthroughput screening methodologies such as microarrays. In addition, numerous databases provide annotation at 
different biological levels. These include databases on the annotation of genes according to the Gene Ontology (GO) nomenclature [1], metabolic pathways as in KEGG [2], or Transcription Factor Binding Sites (TFBS) in TRANSFAC [3] to annotate promoters.

Generally, gene expression data are normalised, filtered and finally genes with similar expression profiles are clustered into groups. The biological hypothesis behind this is that similarly expressed genes have a common biological characteristic, for example participation in the same biological process, or regulation by a common transcription factor.

Several currently available tools provide an interpretation of gene clusters but are often specialised in their analyses. For example, FatiGO [4], GeneLynx [5] and Gostat [6] are powerful tools for GO term identification; GoMiner [7], MAPPFinder [8] and DAVID [9] propose GO and metabolic pathway interpretation; MiCoViTo [10] proposes metabolic pathways and incorporates transcription regulation visualisation; metaSHARK [11] predicts enzymecoding genes from unannotated genome data and places them on generic metabolic pathways; and WebGestalt [12] uses data obtained from different public resources and offers an integrated platform to perform various analyses such as a GO analysis, metabolic pathways and chromosomal distributions.

To facilitate the analysis of gene expression experiments, we have developed MADIBA (MicroArray Data Interface for Biological Annotation), a web based interface with a relational database that currently provides five analytical modules to assist researchers in the identification of possible reasons for the common expression of a cluster of genes. These modules are: (1) a search of over-represented GO terms in the cluster; (2) mapping of the cluster's gene products onto metabolic pathways using the KEGG representation; (3) visualisation of the chromosomal localisation; (4) a search of over-represented motifs in the upstream sequences of the genes and (5) an organism specific analysis.

MADIBA has currently been implemented for Plasmodium falciparum, Oryza sativa (rice) and Arabidopsis thaliana. Malaria is a devastating disease, particularly in Africa, so understanding how its causative agent, Plasmodium, functions is essential. Rice and Arabidopsis are model species for monocotyledonous and dicotyledonous plants respectively [13], and plant analyses are useful particularly for gaining insights into improving crops in both developed and developing countries, for example orphan crops such as cassava, cowpea and pearl millet, which are important for food security in Africa. In addition, Plasmodium is related to plants as the apicoplast (apicomplexan plastid) is reminiscent of the chloroplast [14,15].

\section{Construction and content User interface}

MADIBA is accessible through a simple and user friendly web interface [see Additional file 1]. Once a set of sequences or gene identifiers has been submitted, the user is provided with links to the five analysis modules and the output module. Each analysis module is independent of the others and is accessed individually. In addition, the genes that are to be used in subsequent analyses are listed.

\section{Data submission}

A cluster of genes is submitted to MADIBA, either by uploading a file, or directly pasting a set of nucleotide sequences, in FASTA format. Alternatively, a list of gene identifiers can be submitted. The gene clusters are obtained from any clustering algorithm, such as hierarchical or $k$-means, since MADIBA does not perform any clustering.

For Plasmodium and Arabidopsis sequences, a BLASTN search is performed, and a BLASTX search of the rice sequences is performed to allow the possibility of entering gene clusters from the indica, as well as the japonica, subspecies. In addition, this will potentially allow orthologous gene clusters from other cereals to be analysed, such as pearl millet. Users select which of the BLAST hits they wish to continue the analyses with, and this list of genes is stored.

The gene list is stored for one week on the server, and a unique identifier is provided to allow users to later access and retrieve their data. As each analysis module is accessed, the gene list is used to retrieve the necessary information required by that module from the database. Figure 1 illustrates the architecture and basic data flow of an analysis in MADIBA as described in the next section.

\section{MADIBA modules to analyse gene clusters Gene Ontology module}

This analysis module extracts the GO annotations according to the molecular function, biological process and cellular component ontologies. For a given ontology, a Directed Acyclic Graph (DAG) is drawn in a single view to show the genes from the cluster, and the GO terms that they are annotated to. Each GO term that is found in the cluster is drawn to show its position in the GO hierarchy, in a manner similar to AmiGO's graphical representation [16] and GO::Termfinder [17]. The user is able to select which genes should be visualised, to prevent overly complex graphs. 


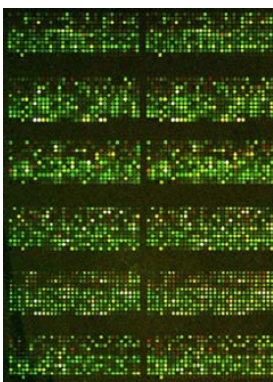

Microarray experiment
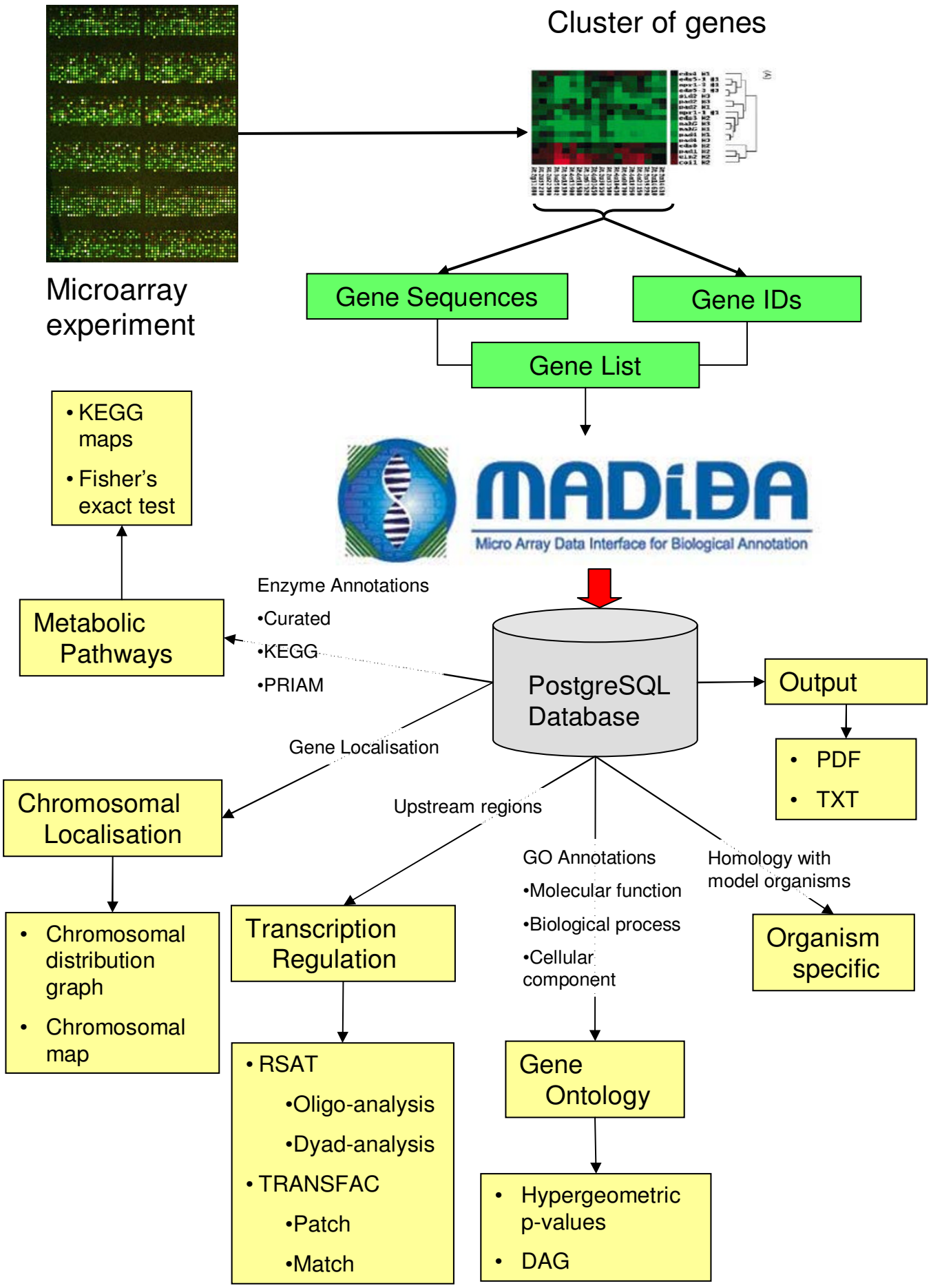

Figure I

A schematic representation of the flow of data through MADIBA. After a microarray experiment, data are normalised and then clustered, since it is hypothesised that the genes in a cluster have common biological implications. A cluster of genes is submitted to MADIBA, either as nucleotide sequences, or gene identifiers. This list of genes can then be subjected to five analysis modules - Gene Ontology Analysis, Metabolic Pathways Analysis, Transcription Regulation Analysis, Chromosomal Localisation Analysis and an Organism Specific Analysis. Also shown are the data that are required by each of the analysis modules. The results from the analyses can be exported as a PDF file, or as plain text. 
An adjusted hypergeometric $p$-value for each annotation is also calculated to evaluate the significance of the annotation. The adjustment methods used are a False Discovery Rate (FDR) adjustment [18], and a Holm adjustment [19], which works under the same assumptions as a Bonferroni correction, but is statistically more powerful [20]. Nodes on the DAG are coloured according to its FDR corrected $p$ value, where red indicates $p$-value $\leq 10^{-10}$; orange, $10^{-10}<p$ value $\leq 10^{-8}$; yellow, $10^{-8}<p$-value $\leq 10^{-6}$; green, $10^{-6}<p$ value $\leq 10^{-4}$; blue, $10^{-4}<p$-value $\leq 10^{-2}$; purple, $p$-value $>$ $10^{-2}$. Other statistical tests will be implemented in the future to provide greater flexibility to the user in calculating the enrichment of the GO terms. A tab delimited text file with each gene and the GO terms associated is provided should users wish to perform their own analyses.

\section{Metabolic Pathways module}

When this module is accessed, a list of all the KEGG metabolic pathways is presented, along with an indication of how many enzymes encoded by genes from the input cluster were found in each pathway. Each pathway in the list is linked to its diagram where the protein products of the genes in the cluster are highlighted. This module compares the enzymatic annotation from three different annotation sources, namely the curated annotation from the original data source (PlasmoDB, TIGR or TAIR); the semi-automatic KEGG annotation and the automatic PRIAM annotation [21]. The use of these diverse and independent annotations increases the robustness of the analysis. Different colours are used to indicate the agreement between the three annotation methods, where yellow indicates that the enzyme was annotated by all three annotation sources; red, by any two annotations; blue, by KEGG only; purple, by PRIAM only; and green, by the original annotators only. In addition, any enzyme found in the genome annotation, but not in the cluster is coloured grey. All elements on the pathways are linked to the KEGG website to provide more information on the particular enzyme or compound. Clicking on an enzyme that is present in the cluster also provides information as to which annotations were used to describe it, and which genes from the cluster encode it.

A $p$-value is calculated for each pathway using Fisher's exact test [22] to indicate the significance of the pathways, using a $2 \times 2$ contingency table where the rows indicate pathway membership and the columns indicate cluster membership [23].

\section{Chromosomal Localisation}

This module permits the identification of co-expressed genes on the same chromosomal region. It provides a bar chart showing the distribution of the genes in the cluster across the chromosomes, that is, the number of genes on each chromosome. In addition, a schematic visualisation of the genes along the chromosomes is provided, where each chromosome is drawn as a horizontal bar, and each gene is represented by a vertical blue line. The size of the bar is relative to the size of the chromosomes, and the genes are drawn in a location with respect to its actual position on the chromosome. Localisation data was obtained from the original annotation source (PlasmoDB, TIGR or TAIR). A mouse-over effect was included to this diagram to allow easier identification of a gene at a particular position.

\section{Transcription Regulation module}

This module presents an approach of motif identification without any prior knowledge, by automatically detecting potential Transcription Factor Binding Sites (TFBS) in the promoter sequences of co-regulated genes, using Regulatory Sequence Analysis Tools (RSAT) [24], specifically using the oligo-analysis and dyad-analysis programs. Oligo-analysis calculates the occurrence of words (oligonucleotides) in a set of sequences, and determines which are overrepresented based on a background model. Dyadanalysis detects overrepresented spaced dyads (oligonucleotide pairs which are separated by a variable spacer region) in a set of sequences. For both analyses, the five most significant motifs are reported, with the option to view all results.

The upstream regions are also searched for known TFBS in the TRANSFAC database (Professional version 11.1) using the built-in Patch and Match programs [25]. Patch uses predefined binding site entries and performs a patternbased binding site search, while Match uses positional weight matrices derived from alignments of binding sites (i.e. matrix-based search). The ten most common motifs found by each tool are presented. For each identified binding site, a link is provided to additional information in TRANSFAC about the binding factor.

\section{Organism Specific Characteristics module}

In the Plasmodium cluster analyses, a component of the aim for this module is to identify putative new drug targets. Thus, a list of the genes without human homologues, with their respective annotations is generated. Also, if any genes similar to the apicoplast are present, its closest homologue to Arabidopsis is identified. Due to its vegetal nature, the apicoplast may provide a target for herbicidelike drugs which will not affect the human host $[14,15]$.

For the plant analyses, the closest Arabidopsis orthologue of each rice gene, and vice versa, is given, in an effort to identify similar genes. This was accomplished by implementing a reciprocal BLASTP search, with a stringent evalue cut-off of $10^{-15}$ to identify highly probable orthologous proteins. In addition, a list of all similar genes, based on sequence similarity is returned, representing the para- 
logues, or protein 'families', for each gene. These results are determined by performing a self BLAST, and the user is able to determine the most relevant results by choosing their own e-value cut-off, minimum percent coverage (how much of the query matched the subject) and minimum percent identity (how much of the match corresponded).

\section{Output}

Results from MADIBA can be exported in either plain text or PDF formats. The user selects the required set of results which is then generated for immediate download.

\section{Implementation}

The MADIBA interface was written in PHP and PythonCGI, and the graphical outputs are dynamically generated using the GD library [26]. A central PostgreSQL database is used to store the downloaded and pre-calculated data.

The downloaded data currently consists of data from the PlasmoDB [27] database for Plasmodium falciparum (release 5.2), TIGR [28] for rice (Oryza sativa ssp japonica cv Nipponbare) (Osa1 database release 5) and TAIR [29] for Arabidopsis thaliana data (TAIR7). We store the gene name, functional annotation, GO identifiers, chromosomal localisations, and the enzymatic annotations (EC identifiers) from the above data sources, as well as the EC numbers proposed by the KEGG Orthology results.

Data pre-calculated by programs before being stored in the database, include putative metabolic enzyme predictions using PRIAM [21]. These predictions are calculated based on enzyme profiles from position specific weight matrices. Also stored are the 1500 nucleotides upstream of the start codon (ATG) based on previous experimental research on Plasmodium promoters [30-33], and 1000 nucleotides upstream of the rice and Arabidopsis genes, as made available by TIGR and TAIR respectively. We also identify putative orthologues between Plasmodium falciparum and human proteins, and rice and Arabidopsis proteins, by performing a reciprocal BLASTP search, with an e-value cut-off of $10^{-15}$. In addition, for rice and Arabidopsis, a BLASTP of all expressed proteins was performed against the proteome of the organism in question (a self BLAST), in order to determine paralogues, or protein 'families', for each gene. The BLAST results with an e-value less than $10^{-3}$ were stored in the database. Sequence information was obtained from the original data source, and BLASTs were performed using a local version of NCBIBLAST.

The DAGs from the GO analysis are drawn using the dot program in Graphviz [34], and visualised using the ZGRViewer applet [35]. All statistical calculations (hypergeometric and Fisher's tests, and multiple hypothesis cor- rections) were computed by the $\mathrm{R}$ statistics package [36], which was accessed using the RPy package [37], a Python interface to R. The KEGG maps are coloured using the Python Imaging Library [38], using the co-ordinates provided by KEGG. The chromosomal distribution bar chart and chromosomal schematic are drawn using the PHP GD library. Both RSAT and TRANSFAC are accessed using system calls. The PDF output files are created using ReportLab [39].

\section{Utility \\ Plasmodium data analysis}

The results of an oligonucleotide array profiling the expression of human and mosquito stages of the malaria parasite's life cycle [40] were chosen to demonstrate the functionalities of MADIBA. After a robust $k$-means clustering algorithm, 15 clusters were proposed, and these clusters were analysed with MADIBA.

After applying MADIBA, an improvement in the number of annotated genes is apparent compared with the original results. The mean fraction of known genes by cluster was $37.5 \%$ compared with $41 \%$ when using MADIBA. The Gene Ontology module automatically allocated annotations to the gene clusters with terms including immune evasion, in cluster 1 , and cell invasion in cluster 15 . In addition, the genes in cluster 2 were correctly identified as involved in gametogenesis and having over-represented protein kinase cascade activity. The metabolic pathways module successfully showed that six of the nine enzymes in the glycolysis pathway were found in cluster 6 , with a $p$ value of 0.04 , as calculated by using Fisher's exact test (Figure 2). This result is further supported by the indication that all the enzymes in the pathway were identified by all three annotation sources, as indicated by the yellow boxes, and by using the GO analysis, it was shown that the anaerobic glycolysis term had a highly significant $p$-value (Figure 3 and Table 1). Using the module specific for Plasmodium characteristics allowed the identification of genes in cluster 3 as interesting drug or vaccine targets, such as PF10_0303, an ookinete surface antigen.

Young et al. [41] performed an analysis on the transcriptome of Plasmodium in an attempt to identify hypothetical genes that are likely to be involved in the sexual development of the parasite. Using an algorithm called ontology-based pattern identification (OPI) on the data, a set of 246 genes were grouped as interesting. Applying RSAT from the Transcriptional Regulation module on this data set identified several overrepresented motifs in the upstream regions of the genes. In particular, oligo-analysis identified the motif GATGAA, which had an expected occurrence of 96.6 based on the background model, but occurred 228 times (E-value for occurrence 10-26). Similarly with dyad analysis, the motif ATCN $\{7\}$ TCA was 


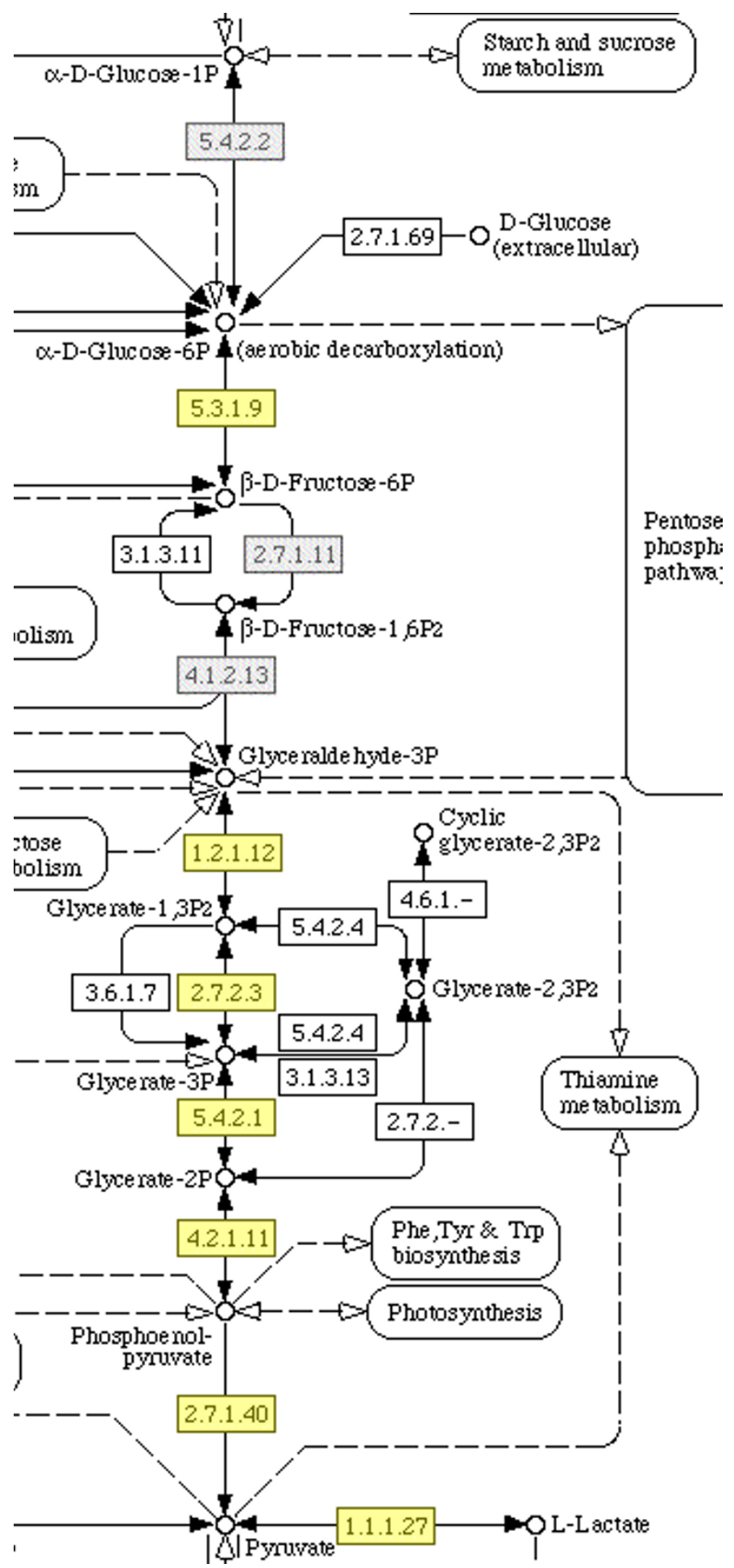

Figure 2

Results from the Metabolic Pathways module. Analysis of cluster 6 of the Plasmodium data [40] revealed that it was noticeably involved in glycolysis. In the KEGG map for glycolysis, it could be seen that almost all of the enzymes involved are present in the cluster. Additionally, all of the enzymes were annotated by all the three annotation sources - the curated annotation from the original data source (PlasmoDB); the semi-automatic KEGG annotation and the automatic PRIAM annotation, as indicated by the yellow boxes. 


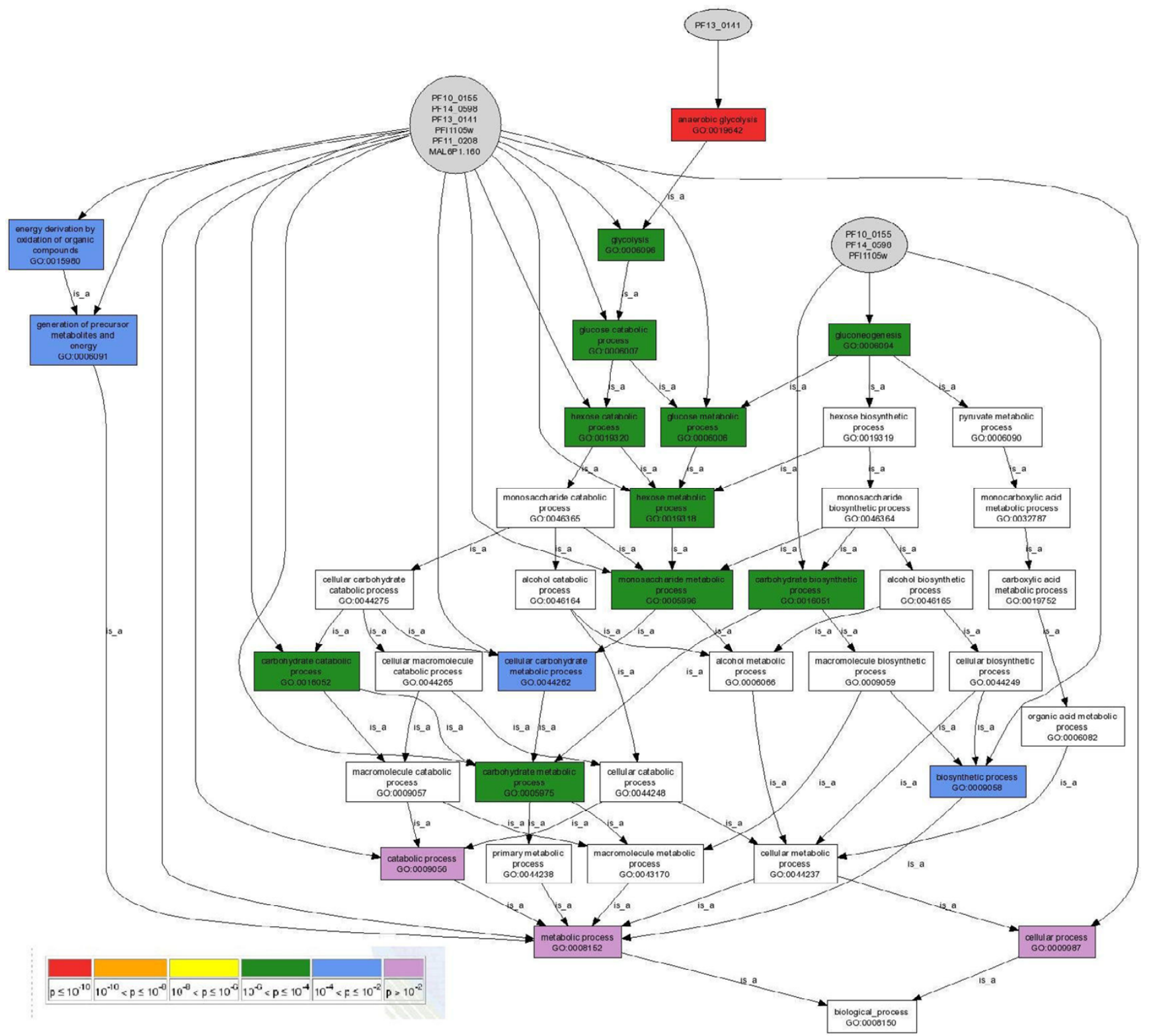

Figure 3

Results from the Gene Ontology module. An analysis of the biological process ontology of the cluster 6 of the Plasmodium data [40] revealed that anaerobic glycolysis was the most significant term. The DAG was reduced to show only the terms that are most relevant to glucose metabolism. The grey ellipses contain the genes that are annotated to the connected GO term and the colour of the GO terms indicates different levels of significance, as indicated by the legend.

found to occur 154 times, as opposed to its expected occurrence of 41.3 (E-value for occurrence $3.5 \times 10-32$ ).

\section{Arabidopsis data analysis}

MADIBA was used to analyse data from a study of the response of Arabidopsis to salt stress [42]. The genes were clustered using a "fuzzy k-means clustering" into 10 major clusters. After analysing individual clusters with MADIBA, it was found that the analyses supported the authors' con- clusions. An example is cluster 0 that had genes responding to osmotic stress in leaves and salt stress in roots, and meta-analysis with other array data by the authors led them to conclude that it contained many biotic stress response genes. Analysis with the Metabolic Pathways module of MADIBA showed over-representation of several enzymes involved with lignin biosynthesis in cluster $0(p=0.001)$ (Figure 4a), which is indicative of a defence response. The Gene Ontology analysis showed enrich- 
Table I: A portion of the table of $p$-values that accompany the DAG (Figure 3) from the analysis of the biological process ontology of cluster 6 of the Plasmodium data [40], showing the p-value calculated from the hypergeometric distribution, along with the Holm and FDR multiple hypothesis corrections.

\begin{tabular}{llccc}
\hline Term & Definition & Corrected p-value (FDR) & Corrected p-value (Holm) & Uncorrected p-value \\
\hline GO:0019642 & anaerobic glycolysis & 0 & 0 & 0 \\
GO:0006096 & Glycolysis & $3.6675 \mathrm{E}-06$ & 0.00010971 & $7.2180 \mathrm{E}-07$ \\
GO:0005996 & monosaccharide metabolic process & $5.3464 \mathrm{E}-06$ & 0.00016747 & $1.1091 \mathrm{E}-06$ \\
GO:0019318 & hexose metabolic process & $5.3464 \mathrm{E}-06$ & 0.00016747 & $1.1091 \mathrm{E}-06$ \\
GO:0006094 & gluconeogenesis & $6.7072 \mathrm{E}-06$ & 0.00021795 & $1.4627 \mathrm{E}-06$ \\
GO:0016051 & carbohydrate biosynthetic process & $6.7072 \mathrm{E}-06$ & 0.00021795 & $1.4627 \mathrm{E}-06$ \\
\hline
\end{tabular}

ment of terms in dihydrocamalexic acid decarboxylase activity, an enzyme responsible for the production of camalexin, a phytoalexin in Arabidopsis produced in response to pathogen infection [43]. Other enriched terms included chitinase activity, ion channel activity, and terms involved in calcium ion activity including ion binding terms and calmodulin binding. The calcium ion responsive terms are most likely related to the effect of salinity on the plant. In the molecular process ontology, terms included regulation of cellular defence responses, hypersensitive response, as well as several biotic stress indicators including responses to ethylene stimulus, jasmonic acid stimulus, salicylic stimulus and abscisic acid stimulus. These hormones are known to be involved in plant defences as well as playing roles in salt-stress signalling, again suggesting cross-talk between the various signalling responses. Cluster 8 was annotated as immediate response genes, and contained members of the WRKY transcription factor family and disease-resistance protein genes. Analysis by the oligo-analysis program of RSAT showed that on the reverse complement, the TTGACT and TTTGAC motifs were overrepresented in the cluster (E-values $1.1 \times 10^{-7}$ and $2 \times 10^{-7}$ respectively), which is similar to the WRKY binding site ((C/T)TGAC(T/C) [44]) (Figure $4 \mathrm{~b})$. In addition, analysis by the TRANSFAC subsection of the Transcription Regulation module showed that a large proportion of the genes ( 110 out of a total of 142 genes in the cluster) contained a motif (ATTTAC) that is functionally important in the promoter of PR-1a, a well characterised pathogenesis related protein [45] (Figure 4c).

\section{Rice data analysis}

For a rice analysis, the results from a cDNA microarray of cultured rice cells in response to flagellin [46] were used. Using $k$-means clustering, 9 clusters were distinguished. The Gene Ontology analysis detected several genes involved in wounding and defence responses. Although the experiment was conducted using the indica subspecies of rice, the results still show an improvement in the annotation and illustrates the ability of the BLASTX search to find orthologous genes.
Additionally, a set of stress-induced rice genes from a transcriptome study on cold, drought, salinity and abscisic acid treatments were tested [47]. While the authors did not perform a statistical clustering, several sets of genes were identified as stress induced and grouped together. After analysing the complete gene set of 73 stress-induced genes with MADIBA, the Gene Ontology analysis identified terms such as responses to stress, cold acclimation, iron ion transport and water deprivation, as being significant, confirming the gene set to be stress responsive. Interestingly, several genes in the set were also annotated as responses to jasmonic- and salicylic-acid, which are usually considered to be signalling molecules in a plant's defence response. This suggests that there is some crosstalk between biotic and abiotic stress responses.

\section{Discussion}

While other tools similar to MADIBA, such as WebGestalt, FatiGO and GoMiner exist, MADIBA differs in that it has a wider range of analyses which can be performed in an integrated fashion, for example, it performs a GO analysis as well as a Transcription Regulation analysis. Of the previously mentioned tools, MADIBA is most similar to WebGestalt [12], which also obtains information from different data sources and provides an integrated set of analysis tools to assist researchers in mining this gene set. WebGestalt, however, does not provide information on transcription regulation, and currently only works for human and mouse data. MADIBA is unique in the organisms it is able to analyse - Plasmodium falciparum, Oryza sativa (rice) and Arabidopsis thaliana. ClutrFree [48] is a desktop-based tool that uses a different approach to facilitate interpretation of microarray data. It is a flexible and generic platform that allows the user to compare different annotation and analysis approaches to a microarray data set. Pattern recognition allows visualisation of the relationships in a directed graph (tree) that assists the user in deriving biological conclusions.

Due to its modular nature, any new analysis can easily be added to MADIBA at a later stage. In addition, the current 
A

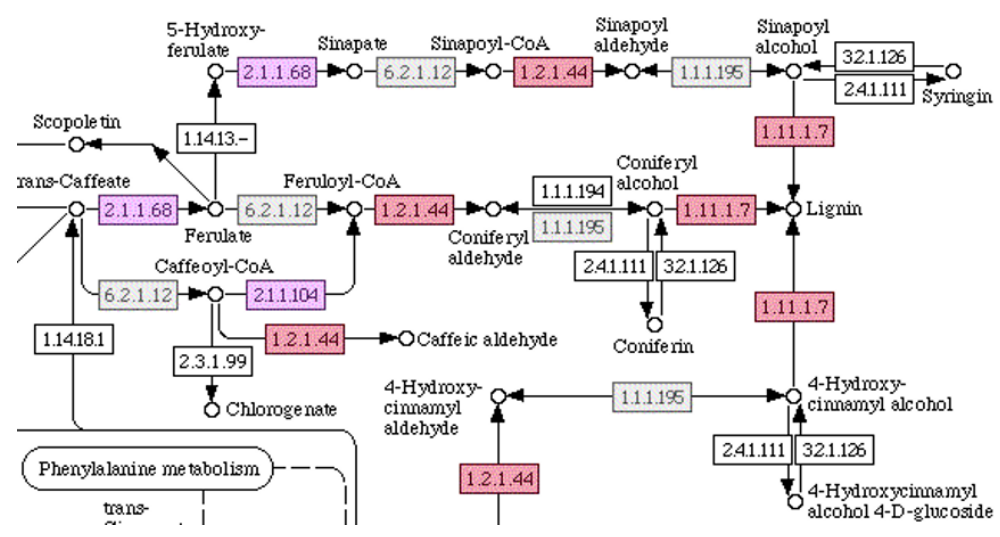

B

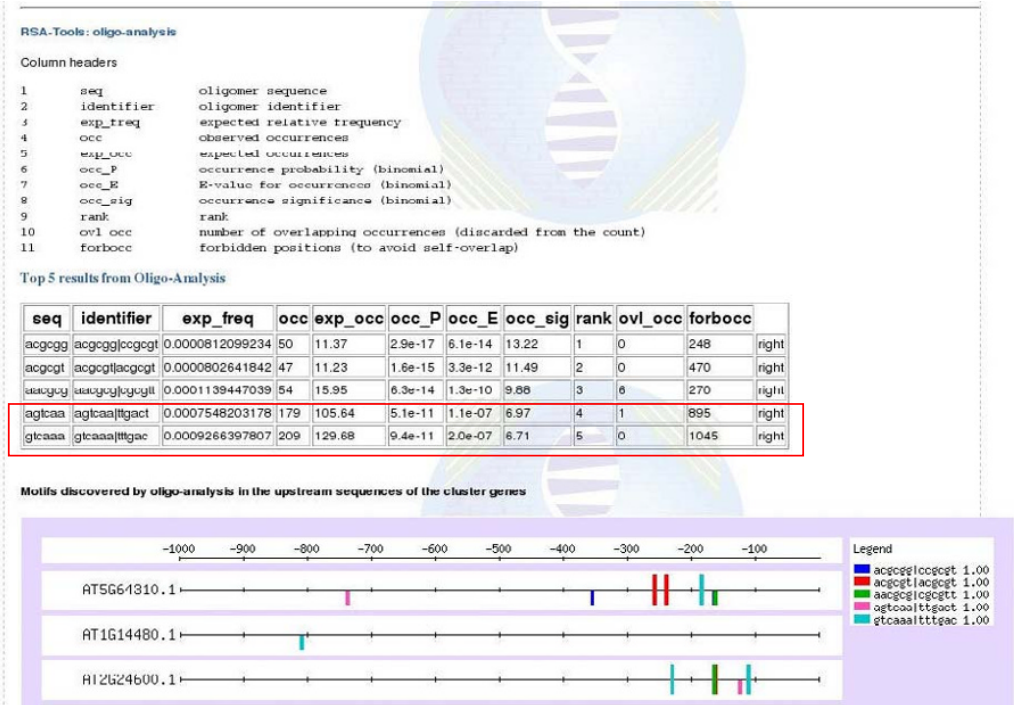

C

\begin{tabular}{|c|c|c|c|c|}
\hline Motif & $\begin{array}{l}\text { TRANSFAC Accession } \\
\text { and ID }\end{array}$ & Description & $\begin{array}{c}\text { Genes } \\
\text { matched }\end{array}$ & $\begin{array}{l}\text { Position, Strand, } \\
\text { Mismatches, Score }\end{array}$ \\
\hline & & & AT3G44260.1 & $75,+0,100.000000$ \\
\hline \multirow[t]{3}{*}{ ATTTAC } & \multirow[t]{3}{*}{$\frac{\text { R11449 }}{\text { NT\$PR1A_04 }}$} & \multirow[t]{3}{*}{$\begin{array}{l}\text { PR-1a (pathogene sis related } \\
\text { prote in 1a): }\end{array}$} & AT1 G42990.1 & $\begin{array}{l}877,+0,100.000000 \\
130,-0,0,100.000000\end{array}$ \\
\hline & & & AT4G17615.2 & $323,-0.100 .000000$ \\
\hline & & & AT2G40000.1 & $\begin{array}{l}766,+, 0,100.000000 \\
488, \sim 0,100.000000\end{array}$ \\
\hline
\end{tabular}

\section{Figure 4}

Results from the Arabidopsis data. (A) Analysis of cluster 0 from the Arabidopsis salt stress experiment [42] with the Metabolic Pathways module revealed that the cluster contained genes involved in lignin biosynthesis. The red colour indicates that the annotations were found by two annotation methods (PRIAM and KEGG in this case), and the purple indicates the enzyme was annotated by PRIAM only. (B) After analysing cluster 8 of the Arabidopsis data [42] with the Transcription Regulation module, it was possible to identify putative transcription factor binding sites. The output of the oligo-analysis tool of RSAT is shown, indicating two motifs on the reverse complement that were identified as similar to the WRKY binding site ((C) T)TGAC(T/C)) (highlighted in the red box). Cluster 8 is known to contain several WRKY transcription factors and several disease-resistance genes. (C) Output from the Patch program of the TRANSFAC sub-module. Shown is the PR-Ia (a pathogenesis related protein) promoter binding site that was identified. The table headers are provided for convenience. 
analysis modules are continually being improved to assist the user in identifying the reasons for the co-expression of a set of genes. Since it is a web application, this makes MADIBA platform-independent and can be accessed from anywhere in the world. Furthermore, the database can be updated, so that the latest information is available to the user. MADIBA is highly dependent on the quality of the genomes' annotations, so as the annotations are improved, so will the results returned by MADIBA.

\section{Future prospects}

All the statistics performed on the data are analysed in terms of the entire genome. However, since whole genome microarray slides are not always used, a proposed improvement is to analyse the data in terms of only the genes that were on the slide.

MADIBA has been designed to be generic and easily expandable, so that any new organisms that are required by the community can readily be incorporated into the database, with only a fully annotated genome necessary. In addition, as the genome annotations are revised, it is important to update the data within MADIBA, and this will be done in a semi-automated manner using pre-built Python scripts.

With the increased number of statistical methods being adopted, we aim to widen the variety of statistical analyses available, such as by including GSEA [49] (through R) or rank tests, to provide a greater level of flexibility for the user.

In the Organism Specific module, orthology was inferred using reciprocal best BLAST hit. We recognise that this is not the most accurate or reliable method for determining orthology, so we are working to implement tools such as Ortholuge [50] and GreenPhyl [51], which take into account phylogenetic information in addition to sequence similarity.

\section{Conclusion}

With the advent of whole genome microarray chips and an increasing number of organisms being sequenced, tools such as MADIBA will become even more significant in understanding the underlying biology. MADIBA provides access to several genomic data sources and analyses, allowing users to quickly annotate and visualise the results. Moreover, this tool can contribute information about Plasmodium as gene regulation in this parasite is poorly understood [52].

\section{Availability and requirements}

MADIBA is freely available and can be accessed on the web using a JavaScript enabled browser at [53]. The home page provides links to an online tutorial, and demonstra- tion gene cluster data and analysis results, including those discussed above.

\section{Authors' contributions}

PJL was involved in expanding MADIBA to include the plant data, improving the metabolic pathways and GO modules, and prepared the manuscript. CCR initiated the project, was responsible for the Plasmodium data, and drafted the manuscript. FJ was responsible for bioinformatics and participated in its design. AIL conceived the study and participated in its design. DKB was involved in plant analyses, participated in its design and preparation of the manuscript. All authors have read and approved the manuscript.

\section{Additional material}

\section{Additional file 1}

MADIBA initial submission page. A screen shot of MADIBA after a set of sequences has been submitted. Block A illustrates the links to the five analysis modules and the output module, Block B shows the unique identifier that is provided to the user and section $C$ lists the genes that are to be used in subsequent analyses.

Click here for file

[http://www.biomedcentral.com/content/supplementary/14712164-9-105-S1.jpeg]

\section{Acknowledgements}

The MADIBA project is funded by the University of Pretoria, the NRF (National Research Foundation) and the NBN (National Bioinformatics Network) of South Africa. The postdoctoral fellowship was funded by the University of Pretoria (CCR) and PJL received a bursary from the NBN. We are grateful to Jeremy Baxter for advice on the statistical analyses.

\section{References}

I. Ashburner M, Ball CA, Blake JA, Botstein D, Butler H, Cherry JM, Davis AP, Dolinski K, Dwight SS, Eppig JT, Harris MA, Hill DP, IsselTarver L, Kasarskis A, Lewis S, Matese JC, Richardson JE, Ringwald M, Rubin GM, Sherlock G: Gene ontology: tool for the unification of biology. The Gene Ontology Consortium. Nat Genet 2000, 25:25-29.

2. Kanehisa M, Goto S, Kawashima S, Okuno Y, Hattori M: The KEGG resource for deciphering the genome. Nucleic Acids Res 2004, 32:D277-D280.

3. Matys V, Fricke E, Geffers R, Gossling E, Haubrock M, Hehl R, Hornischer K, Karas D, Kel AE, Kel-Margoulis OV, Kloos DU, Land S, Lewicki-Potapov B, Michael H, Munch R, Reuter I, Rotert S, Saxel H, Scheer M, Thiele S, Wingender E: TRANSFAC(R): transcriptional regulation, from patterns to profiles. Nucl Acids Res 2003, 31:374-378.

4. Al Shahrour F, Diaz-Uriarte R, Dopazo J: FatiGo: a web tool for finding significant associations of Gene Ontology terms with groups of genes. Bioinformatics 2004, 20:578-580.

5. Lenhard B, Hayes WS, Wasserman WW: GeneLynx: A GeneCentric Portal to the Human Genome. Genome Res 200I, I I:2I5I-2I57.

6. Beissbarth T, Speed TP: GOstat: find statistically overrepresented Gene Ontologies within a group of genes. Bioinformatics 2004, 20: I464- I 465.

7. Zeeberg BR, Feng W, Wang G, Wang MD, Fojo AT, Sunshine M, Narasimhan S, Kane DW, Reinhold WC, Lababidi S, Bussey KJ, Riss J, Barrett JC, Weinstein JN: GoMiner: a resource for biological 
interpretation of genomic and proteomic data. Genome Biol 2003, 4:R28.

8. Doniger SW, Salomonis N, Dahlquist KD, Vranizan K, Lawlor SC, Conklin BR: MAPPFinder: using Gene Ontology and GenMAPP to create a global gene-expression profile from microarray data. Genome Biol 2003, 4:R7.

9. Dennis G, Sherman B, Hosack D, Yang J, Gao W, Lane H, Lempicki R: DAVID: Database for Annotation, Visualization, and Integrated Discovery. Genome Biol 2003, 4(5):3. Epub 2003 Apr 3.

10. Lelandais G, Marc P, Vincens P, Jacq C, Vialette S: MiCoViTo: a tool for gene-centric comparison and visualization of yeast transcriptome states. BMC Bioinformatics 2004, 5:20.

II. Pinney JW, Shirley MW, McConkey GA, Westhead DR: metaSHARK: software for automated metabolic network prediction from DNA sequence and its application to the genomes of Plasmodium falciparum and Eimeria tenella. Nucl Acids Res 2005, 33:1399-1409.

12. Zhang B, Kirov S, Snoddy J: WebGestalt: an integrated system for exploring gene sets in various biological contexts. Nucleic Acids Res 2005, 33:W74I-W748.

13. Rensink WA, Buell CR: Arabidopsis to rice. Applying knowledge from a weed to enhance our understanding of a crop species. Plant Physiol 2004, I 35:622-629.

14. Marechal E, Cesbron-Delauw MF: The apicoplast: a new member of the plastid family. Trends in Plant Science 200I, 6:200-205.

15. Ralph SA, D'Ombrain MC, McFadden Gl: The apicoplast as an antimalarial drug target. Drug Resist Updat 200I, 4:145-I5I.

16. AmiGO 2007 [http://amigo.geneontology.org/cgi-bin/amigo/go.cgi].

17. Boyle El, Weng S, Gollub J, Jin H, Botstein D, Cherry JM, Sherlock G: GO::TermFinder--open source software for accessing Gene Ontology information and finding significantly enriched Gene Ontology terms associated with a list of genes. Bioinformatics 2004, 20:3710-37I5.

18. Benjamini Y, Hochberg Y: Controlling the False Discovery Rate: A Practical and Powerful Approach to Multiple Testing. J Roy Stat Soc 1995, 57:289-300.

19. Holm S: A simple sequentially rejective multiple test procedure. Scandinavian Journal of Statistics 1979, 6:65-70.

20. Aickin $M$, Gensler $H$ : Adjusting for multiple testing when reporting research results: the Bonferroni vs Holm methods. Am J Public Health 1996, 86:726-728.

21. Claudel-Renard C, Chevalet C, Faraut T, Kahn D: Enzyme-specific profiles for genome annotation: PRIAM. Nucl Acids Res 2003, 31:6633-6639.

22. Fisher RA: The logic of inductive inference. J Roy Stat Soc 1935 , 98:39-82.

23. Chung HJ, Kim M, Park CH, Kim J, Kim JH: ArrayXPath: mapping and visualizing microarray gene-expression data with integrated biological pathway resources using Scalable Vector Graphics. Nucleic Acids Res 2004, 32:W460-W464.

24. van Helden J: Regulatory sequence analysis tools. Nucleic Acids Res 2003, 31 1:3593-3596.

25. Matys V, Kel-Margoulis OV, Fricke E, Liebich I, Land S, Barre-Dirrie A, Reuter I, Chekmenev D, Krull M, Hornischer K, Voss N, Stegmaier $P$, Lewicki-Potapov B, Saxel $\mathrm{H}$, Kel AE, Wingender E: TRANSFAC and its module TRANSCompel: transcriptional gene regulation in eukaryotes. Nucleic Acids Res 2006, 34:DI 08-DI 10.

26. GD Graphics Library 2007 [http://www.boutell.com/gd].

27. Fraunholz MJ, Roos DS: PlasmoDB: exploring genomics and post-genomics data of the malaria parasite, Plasmodium falciparum. Redox Rep 2003, 8:317-320.

28. Yuan Q, Ouyang S, Wang A, Zhu W, Maiti R, Lin H, Hamilton J, Haas B, Sultana R, Cheung F, Wortman J, Buell CR: The Institute for Genomic Research Osal Rice Genome Annotation Database. Plant Physiol 2005, I38:18-26.

29. Rhee SY, Beavis W, Berardini TZ, Chen G, Dixon D, Doyle A, GarciaHernandez M, Huala E, Lander G, Montoya M, Miller N, Mueller LA, Mundodi S, Reiser L, Tacklind J, Weems DC, Wu Y, Xu I, Yoo D, Yoon J, Zhang P: The Arabidopsis Information Resource (TAIR): a model organism database providing a centralized, curated gateway to Arabidopsis biology, research materials and community. Nucleic Acids Res 2003, $31: 224-228$.

30. Crabb BS, Cowman AF: Characterization of promoters and sta ble transfection by homologous and nonhomologous recombination in Plasmodium falciparum. Proc Natl Acad Sci U S A 1996, 93:7289-7294.
31. Dechering KJ, Kaan AM, Mbacham W, Wirth DF, Eling W, Konings $\mathrm{RN}$, Stunnenberg HG: Isolation and functional characterization of two distinct sexual-stage-specific promoters of the human malaria parasite Plasmodium falciparum. Mol Cell Biol 1999, 19:967-978.

32. Horrocks P, Dechering K, Lanzer M: Control of gene expression in Plasmodium falciparum. Mol Biochem Parasitol 1998 95:17|-|8|

33. Lanzer $M$, de Bruin $D$, Ravetch JV: A sequence element associated with the Plasmodium falciparum KAHRP gene is the site of developmentally regulated protein-DNA interactions. Nucleic Acids Res 1992, 20:305 I-3056.

34. Graphviz - Graph Visualization Software 2007 [http:// www.graphviz.org/]

35. ZGRViewer 2007 [http://zvtm.sourceforge.net/zgrviewer.html]

36. R Programming Language 2007 [http://www.r-project.org/].

37. RPy 2008 [http://rpy.sourceforge.net/].

38. Python Imaging Library 2008 [http://www.pythonware.com/ products/pil/].

39. ReportLab 2007 [http://www.reportlab.org/].

40. Le Roch KG, Zhou Y, Blair PL, Grainger M, Moch JK, Haynes JD, De la Vega P, Holder AA, Batalov S, Carucci DJ, Winzeler EA: Discovery of Gene Function by Expression Profiling of the Malaria Parasite Life Cycle. Science 2003, 30 I:I503-I508.

4I. Young JA, Fivelman QL, Blair PL, de la Vega P, Le Roch KG, Zhou Y, Carucci DJ, Baker DA, Winzeler EA: The Plasmodium falciparum sexual development transcriptome: a microarray analysis using ontology-based pattern identification. Mol Biochem Parasitol 2005, | 43:67-79.

42. $\mathrm{Ma} \mathrm{S}$, Gong $\mathrm{Q}$, Bohnert $\mathrm{HJ}$ : Dissecting salt stress pathways. J Exp Bot 2006, 57:1097-II07.

43. Nafisi M, Goregaoker S, Botanga CJ, Glawischnig E, Olsen CE, Halkier $\mathrm{BA}$, Glazebrook J: Arabidopsis cytochrome P450 monooxygenase 7|A|3 catalyzes the conversion of indole-3-acetaldoxime in camalexin synthesis. Plant Cell 2007, 19:2039-2052.

44. Ulker B, Somssich IE: WRKY transcription factors: from DNA binding towards biological function. Current Opinion in Plant Biology 2004, 7:49I-498.

45. Buchel AS, Brederode FT, Bol JF, Linthorst HJ: Mutation of GT-I binding sites in the Pr-IA promoter influences the level of inducible gene expression in vivo. Plant Mol Biol 1999, 40:387-396.

46. Fujiwara S, Tanaka N, Kaneda T, Takayama S, Isogai A, Che FS: Rice cDNA microarray-based gene expression profiling of the response to flagellin perception in cultured rice cells. Mol Plant Microbe Interact 2004, 17:986-998.

47. Rabbani MA, Maruyama K, Abe H, Khan MA, Katsura K, Ito Y, Yoshiwara K, Seki M, Shinozaki K, Yamaguchi-Shinozaki K: Monitoring Expression Profiles of Rice Genes under Cold, Drought, and High-Salinity Stresses and Abscisic Acid Application Using cDNA Microarray and RNA Gel-Blot Analyses. Plant Physiol 2003, 133:1755-1767.

48. Bidaut G, Ochs MF: ClutrFree: cluster tree visualization and interpretation. Bioinformatics 2004, 20:2869-287I.

49. Subramanian A, Tamayo P, Mootha VK, Mukherjee S, Ebert BL, Gillette MA, Paulovich A, Pomeroy SL, Golub TR, Lander ES, Mesirov JP: From the Cover: Gene set enrichment analysis: A knowledge-based approach for interpreting genome-wide expression profiles. Proceedings of the National Academy of Sciences 2005, 102(43): I 5545-I5550. Epub 2005 Sep 30

50. Fulton DL, Li YY, Laird MR, Horsman BG, Roche FM, Brinkman FS: Improving the specificity of high-throughput ortholog prediction. BMC Bioinformatics 2006, 7:270.

51. Conte MG, Gaillard S, Lanau N, Rouard M, Perin C: GreenPhyIDB: a database for plant comparative genomics. Nucleic Acids Res 2008:D99/-8. Epub 2007 Nov 5.

52. Daily JP, Scanfeld D, Pochet N, Le Roch K, Plouffe D, Kamal M, Sarr O, Mboup S, Ndir O, Wypij D, Levasseur K, Thomas E, Tamayo P, Dong C, Zhou Y, Lander ES, Ndiaye D, Wirth D, Winzeler EA, Mesirov JP, Regev A: Distinct physiological states of Plasmodium falciparum in malaria-infected patients. In Nature Volume 450. Nature Publishing Group; 2007:1091-1095

53. MADIBA 2007 [http://www.bi.up.ac.za/MADIBA] 